\title{
Interacciones y consecuencias del consumo combinado de alcohol y cocaína: una actualización sobre el cocaetileno'
}

\author{
D. Raul Pastor*, Juan José llopis**, D. Abel Baquero*** \\ *Lab. Psicobiología, Universitat Jaume I. **Área de Psicobiología, Universitat Jaume I \\ *** Centro de Encuentro y Acogida, Fundacio Salut i Comunitat
}

\section{RESUMEN}

Los pacientes que solicitan tratamiento por consumo de cocaína y abuso de alcohol, presentan peculiaridades respecto de los que sólo consumen cocaína. La ingesta de alcohol como detonante del "craving" y conducta de búsqueda compulsiva de cocaína, influye en haber una mayor perdida de control del consumo, más problemas sociales, más conductas de riesgo y antisociales.

Hipótesis: la presencia de un metabolito denominado Cocaetileno, resultante del consumo simultaneo de alcohol y cocaína, podría explicar, la mayor toxicidad y compulsividad de estos episodios.

Metodología: Se realiza una revisión de la literatura científica sobre las consecuencias de la interacción alcohol-cocaína.

Resultados: La interacción metabólica alcohol-cocaína incrementa el potencial tóxico de ambas sustancias por separado.

El cocaetileno actúa como tóxico per se. Su presencia en el organismo provoca mayor riesgo potencial en los consumo simultáneos de alcohol y cocaína.

Existen concentraciones de cocaetileno más significativas cuando el alcohol se administra previamente a la cocaína.

Los resultados de la investigación básica muestran que, gran parte de las diferencias observadas en la acción de ambas sustancias, cuando se ingieren conjuntamente, pudieran estar debidas a modificaciones en la farmacocinética de dichas drogas y a la potencial acción del cocaetileno lo que, sumado a las respectivas acciones del alcohol y la cocaína, podría ser la base de la mayor gravedad de los cuadros clínicos observados.

Palabras clave: Cocaína, Alcohol, Cocaetileno, Toxicidad, Abuso.

\section{ABSTRACT}

Patients who request treatment for cocaine use and alcohol abuse present differences from those who use cocaine only. Ingestion of alcohol as a detonator of craving and the compulsive search behaviour of cocaine leads to a greater loss of control on use, more social problems, more risk and more antisocial behaviours.

Hypothesis: the presence of a metabolite known as cocaethylene, resulting from the simultaneous use of alcohol and cocaine could explain the greater toxicity and compulsivity of these episodes.

Methodology: A review was made of the scientific literature on the consequences of alcohol-cocaine interaction.

Results: The alcohol-cocaine metabolic interaction increases the potential toxicity of both substances taken separately.

The cocaethylene acts as a toxic per se. Its presence in the organism provokes a higher potential risk in the simultaneous consumption of alcohol and cocaine.

There are more significant concentrations of cocaethylene when alcohol is administered prior to the cocaine.

The results of the basic research show that a large part of the differences observed in the action of both substances, when ingested simultaneously, could be a result of modifications in the pharmacokinetics of said drugs and of the potential action of the cocaethylene, which, added to the respective actions of alcohol and cocaine, could be the basis of the increased severity observed in clinical profiles.

Key words: Cocaine, Alcohol, Cocaethylene, Toxicity, Abuse.

\section{INTRODUCCIÓN}

Las tendencias en el consumo de drogas en Europa se incrementan para el consumo de cocaína. España y los Países Bajos presentan el mayor consumo de cocaína de Europa, entre el 1 y el $4 \%$ de la población general. En Estados Unidos la incidencia todavía es mayor ya que alrededor del 8\% de la población general ha consumido cocaína en alguna ocasión. (O.E.D.T., 2001).

Entre 1996 y 1999 las admisiones a tratamiento por cocaína aumentaron en un 187\%. De estas perso- 
nas que iniciaron tratamiento en ese período de tiempo cerca de un $65 \%$ consumía conjuntamente alcohol. En 1999 la cocaína fue la droga que en más ocasiones se relacionó con los episodios de urgencias 39,4 \% (P.N.D., 2001).

En Estados Unidos, unos 12 millones de personas padecen algún tipo de dependencia o abuso de cocaína y alcohol (Wilson et al., 2001).

Algunos estudios sobre percepción de riesgo y tendencias en población general comienzan a mostrar las peculiaridades del uso de cocaína. Todo parece indicar que el uso de cocaína y alcohol se complementa dentro de la escena de consumo. En el estudio sobre "la vida social de la cocaína" (Calafat et al., 2001), la practica totalidad de los consumidores entrevistados vinculan el alcohol y la cocaína, sugiriendo una cierta "obligatoriedad" en el consumo para poder controlar los efectos de ambas.

Estos datos apuntan, de forma significativa, que en los últimos años se está produciendo un agravamiento en las consecuencias biopsicosociales del consumo de cocaína y alcohol, por lo que pensamos que debe existir algún fenómeno que explique esta evolución.

En estos momentos nos encontramos con que ya han aparecido a nivel asistencial las repercusiones del consumo abusivo de cocaína y alcohol de forma que en los últimos años venimos observando un grupo de pacientes, cada vez más amplio, que solicitan tratamiento por problemas con la cocaína y que, simultáneamente, realizan consumos abusivos de alcohol. Prácticamente en la totalidad de los casos al realizar una evaluación posterior a la intervención inicial suelen ser diagnosticados, también, de abuso/dependencia de alcohol.

Este grupo de pacientes presenta peculiaridades que les diferencian de aquellos que consumen cocaína aisladamente y que se caracterizan por:

- Mayor percepción de control sobre el consumo que en otras adicciones. En especial en lo que se refiere a la ingesta de alcohol.

- Percepción de problemas con la cocaína pero no con el alcohol. En cambio su historia de consumo lleva al diagnóstico de abuso/dependencia de alcohol.

- Progresivo incremento en las cantidades consumidas en cada episodio, pero no tanto en la frecuencia de los episodios de consumo.

- La ingesta de alcohol aparece como detonante del craving y conducta de búsqueda compulsiva de cocaína.

- Mayor perdida de control del consumo comparado con sujetos que no asocian cocaína y alcohol.

- Aparición de conductas con rasgos antisociales tras el consumo.
- Mayores consecuencias sociales, laborales, familiares y conductuales con incremento de las conductas de riesgo a nivel sexual, grandes gastos, ludopatía, peleas, problemas de pareja y separación, frecuentar ambientes de prostitución, sexo compulsivo y relaciones sin protección.

\section{OBJETIVO}

Valorar la especial peculiaridad de la ingesta conjunta de alcohol y cocaína mediante el conocimiento de la influencia del producto metabólico de ambos, denominado cocaetileno, sobre el cuadro de abuso/ dependencia a estas drogas.

Para la formulación del objetivo de este trabajo nos basamos en la observación clínica de la presencia de peculiaridades en los pacientes con consumos de abuso simultáneos de alcohol y cocaína, y más concretamente:

- El consumo simultaneo de alcohol y cocaína tiene mayores consecuencias que el consumo de estas dos drogas por separado.

- La ingesta de alcohol provoca incremento del craving de cocaína con mayor gravedad en los episodios de consumo, mayor perdida de control e intoxicación más grave.

- La presencia de un metabolito denominado cocaetileno, resultante de la interacción por ingesta simultanea de alcohol y cocaína, podría explicar la mayor toxicidad y compulsividad de estos episodios de consumo.

\section{METODOLOGÍA}

Revisión de publicaciones científicas referidas a las consecuencias derivadas de la interacción entre alcohol y cocaína, y en particular sobre las acciones del cocaetileno.

\section{RESULTADOS}

Interacción farmacológica alcohol-cocaína.

La cocaína es rápidamente metabolizada por hidrólisis de su grupo metil-ester en benzoilecgonina, metabolito desprovisto de propiedades psicoestimulantes o psicomotoras (Spealman et al., 1989).

En preparaciones in vitro de tejido hepático humano, se han identificado carboxilesterasas capaces de hidrolizar el grupo metil-ester de la cocaína (Dean et al., 1991). El etanol produce una inhibición de este paso metabólico, disminuyendo la hidrólisis de cocaí- 
na a benzoilecgonina (Brzezinski et al., 1994). En presencia de etanol, estas carboxilesterasas catalizan la cocaína a su homólogo etílico: el Cocaetileno o etilcocaína (Dean et al., 1991).

\section{Figura 1. Paso metabólico de producción de Cocaetileno o etilcocaína}

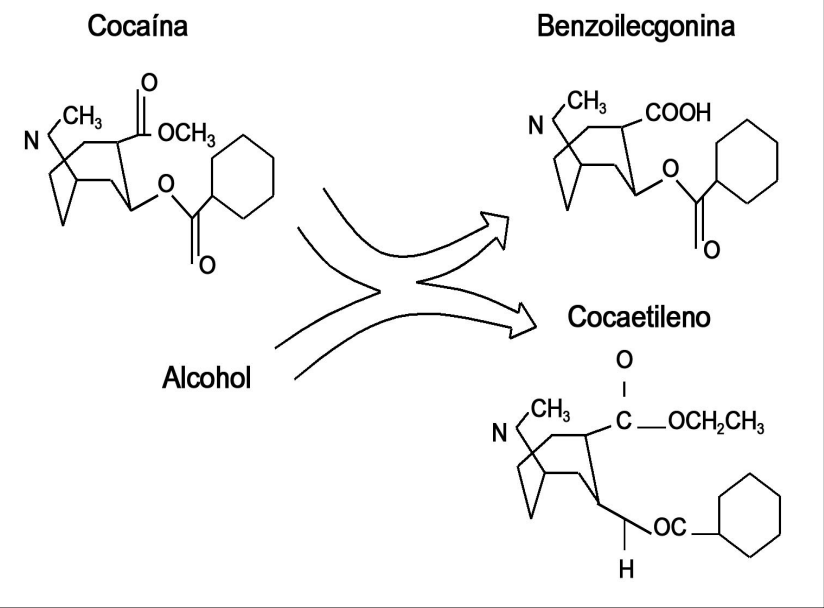

Paralelamente, en estudios ex vivo con roedores cotratados con alcohol y cocaína, se ha constatado la detección de cocaetileno en suero y en todos los tejidos analizados (Dean et al., 1997). Estos hallazgos sugieren importantes modificaciones en la biotransformación y eliminación de la cocaína derivadas de la interacción metabólica alcohol-cocaína.

Tras la coexposición alcohol-cocaína, el cocaetileno se encuentra rápida y principalmente en hígado, pulmón y riñón. Unos minutos más tarde, este metabolito es detectado en otros tejidos como el cerebro, el corazón o el bazo. Esta distribución temporal sugiere que la formación del cocaetileno ocurriría principalmente en el hígado para posteriormente ser distribuido por el resto del organismo (Dean et al., 1997).

Principalmente, la previa administración aguda de alcohol, parece reducir los niveles de benzoilecgonina, prologándose, así, la detección de cocaína en sangre (Dean et al., 1997). En el caso de un pretratamiento crónico de alcohol en roedores, también se observa un incremento significativo de las concentraciones de cocaína y alcohol, tanto en sangre como en orina, respecto a animales control (Pan y Hedaya, 1999; Farré et al., 1993).

En estudios realizados con voluntarios humanos y bajo condiciones experimentales controladas, una vez administradas las dos sustancias, se detectó cocaetileno en suero y plasma de manera dependiente de la dosis de etanol ( Perez-Reyes y Jeffcoat, 1992; Farré et al., 1993). Uno de los factores más relevantes parece ser la influencia del orden de administración de ambas drogas. Así, las concentraciones de cocaetileno más significativas se observan cuando el alcohol se administra previamente a la cocaina $y$, en cambio, deja de evidenciarse si se invierte este orden de administración (McCance-Katz et al., 1991; Perez-Reyes, 1994). A su vez, también se ha identificado cocaetileno en orina, sangre, cerebro e hígado, obtenidos en autopsias de individuos que habían ingerido ambas sustancias y en muestras de sangre tomadas a pacientes atendidos en servicios de urgencias hospitalarios (Jatlow et al., 1996; Hearn et al., 1991).

\section{Cocaetileno: Acciones en el SNC.}

A diferencia de la cocaína, el cocaetileno parece mostrar una menor afinidad por los transportadores de serotonina, siendo más selectivo para la dopamina (Baumann et al., 1998)

Al igual que la cocaína, el cocaetileno interfiere en la recaptación presináptica de dopamina (Hearn et al., 1991) lo que se traduce en un incremento en la concentración extracelular de dopamina en núcleo accumbens o en núcleo caudado de roedores (Lindholm et al., 2001). También en primates, se ha constatado que el cocaetileno incrementa los niveles extracelulares de dopamina en el núcleo caudado (lyer et al., 1995).

La coadministración de alcohol y cocaína, eleva los niveles citoplasmáticos de b-endorfinas en el núcleo arqueado hipotalámico de ratas, de forma muy superior al conseguido por una administración de cocaína (Hayase et al., 1998).

En esta misma línea, se ha demostrado la liberación de hormona adrenocorticotropa y corticoesterona en respuesta a una administración de cocaetileno en el núcleo paraventricular, implicando por tanto al eje hipotalámico-pituitario-adrenal (Torres et al., 1996).

Estas evidencias han sido completadas en paradigmas conductuales. La administración de cocaetileno en roedores es capaz de :

- Incrementar la actividad motora (Baumann et al., 1998).

- Promover el establecimiento de patrones instrumentales de autoadministración (Landry, 1992).

- Producir sensibilización cruzada con la cocaína (Horger et al., 1996).

- Sensibilización dependiente de contexto (Prinssen et al., 1996).

- Preferencia de lugar condicionada (Schechter, 1995).

- Inducir mayores propiedades reforzantes que la cocaína por si sola (Raven et al., 2000).

Estos hallazgos refuerzan la hipótesis de una potencial acción del cocaetileno sobre el sistema nervioso central, que se sumaria a las respectivas acciones del alcohol y cocaína sobre el mismo sustrato neural. 


\section{Toxicología.}

La interacción alcohol-cocaína interfiere en el patrón de degradación tanto del alcohol (Farré et al., 1993) como de la cocaína (Pan y Hedaya, 1999), incrementando, de esta forma, el potencial tóxico de ambas sustancias.

La presencia en el organismo de cocaetileno se ha podido asociar con convulsiones, cardiotoxicidad, daño hepático, repercusiones en el sistema inmunitario y letalidad. (Pirozhkov et al., 1993; Andrews, 1997).

Afecta la contractilidad del miocardio en mayor medida que la cocaína (Qiu y Morgan, 1993). El cocaetileno se ha mostrado un potente bloqueador de los canales de sodio en el miocardio (Xu et al., 1994) que prolonga la cardiotoxicidad en comparación con la de ambas drogas por separado. Asimismo, los picos en suero de cocaetileno parecieron asociarse con una depresión miocardial prolongada (Wilson et al., 2001).

A nivel hepático se incrementa la toxicidad de la cocaína cuando previamente se administra alcohol (Jover et al., 1991). En algunos estudios se han descrito lesiones concretas del tipo necrosis centrolobulillar con la particularidad de ser dosis dependiente de cocaetileno (Roberts et al., 1992).

En estudios de letalidad se ha evidenciado el elevado poder tóxico del cocaetileno. La dosis letal (LD50) de cocaetileno es significativamente inferior que la de la cocaína (Schechter y Meehan, 1995). El riesgo potencial de muerte súbita por consumo de alcohol y cocaína se incrementa de 18 a 25 veces respecto al de la cocaína, según los diferentes estudios (Rose et al., 1990; Andrews, 1997)

Aunque no hay muchas investigaciones que profundicen en la clínica derivada del consumo de alcohol y cocaína en sujetos consumidores/adictos o en voluntarios, a los datos de experimentación animal ya señalados, pueden sumarse los referentes a investigaciones de carácter epidemiológico, relativas a atención médica tras el consumo de alcohol y cocaína.

En este sentido, el uso/abuso de ambas sustancias se ha relacionado con déficits cognitivos en memoria, atención, orientación y asimetrías sensoriales. Además, se han observado deterioros más marcados a medida que avanza el consumo, llegando a imposibilitar el adecuado funcionamiento del sujeto (Singer et al., 2000).

En comparación con las intoxicaciones por cocaína, los pacientes atendidos en servicios de urgencias por consumo de alcohol y cocaína, presentaban cuadros clínicos más graves, tasa cardiaca y presión arterial más elevada, estado mental más deteriorado, así como mayor frecuencia de intubaciones y mayor severidad del tratamiento requerido (Vanek et al., 1996; Signs et al., 1996).
Pensamos que las evidencias mostradas en las diferentes investigaciones básicas revisadas en este texto vienen a apoyar la evidencia clínica observada entre los adictos que realizan consumos de abuso simultáneos de alcohol y cocaína y que se manifiestan por un mayor deterioro, con consecuencias más graves a todos los niveles. Esta tendencia en las pautas de consumo, pensamos, que se va generalizando entre los consumidores.

Por nuestra parte hemos iniciado el planteamiento de intervenciones farmacológicas dirigidas a evitar esta potenciación de los efectos del consumo, para lo que ya estamos valorando el uso de fármacos aversivos del alcohol pautados bajo consentimiento informado y también la posibilidad de la utilización de fármacos antagonistas opiáceos que limiten el consumo de alcohol y eviten la perdida de control una vez iniciada la ingesta, como puede ser el caso de tratamientos farmacológicos con naltrexona para reducir el "priming" y limitar las consecuencias de la ingesta de ambas sustancias adictivas.

\section{CONCLUSIONES}

La interacción metabólica alcohol-cocaína incrementa el potencial tóxico de ambas sustancias por separado.

El cocaetileno actúa como un producto tóxico per se a todos los niveles.

La presencia del producto metabólico cocaetileno provoca mayor riesgo potencial en los consumo simultáneos de alcohol y cocaína.

Las concentraciones de cocaetileno más significativas se observan cuando el alcohol se administra previamente a la cocaína.

De estos hallazgos científicos parece derivarse que, gran parte de las diferencias observadas en cuanto a la acción de ambas sustancias, cuando se toman conjuntamente, pudieran estar debidas a modificaciones en la farmacocinética de dichas drogas y a la potencial acción del cocaetileno, sumada a las respectivas acciones del alcohol y la cocaína, pasando a ser la base de los cuadros clínicos de mayor gravedad observados en estos adicto

Existen múltiples datos en la investigación básica tanto en animales como en humanos que apoyan la formulación de nuestras sospechas clínicas y permiten la formulación de nuevas hipótesis en relación a las manifestaciones clínicas que de este consumo simultáneo se observan.

No obstante es totalmente necesario comprobar las hipótesis que se desprenden de la literatura revisada, mediante estudios clínicos que permitan conocer la situación real y de esta forma poder abordar con 
mayores garantías las demandas asistenciales provocadas por el abuso/dependencia de alcohol y cocaína.

\section{BIBLIOGRAFÍA}

Andrews, P. (1997). Cocaethylene toxicity. J Addict Dis 16: 75-84.

Baumann, M.H.; Horowitz, J.M.; Kristal, M.B. and Torres, G. (1998). Effects of cocaethylene on dopamine and serotonin synthesis in Long-Evans and Sprague Dawley brains. Brain Research 804: 316-319.

Brzezinski, M.R.; Abraham, T.L.; Stone, C.L.; Dean, R.A. and Bosron, W.F. (1994). Purification and characterization of a human liver cocaine carbolylesterase that catalyzes the production of benzoylecgonine and the formation of cocaethylene from alcohol and cocaine. Biochem Pharmacol 48: 1747-1755.

Calafat, A.; Juan, M.; Becoña, E.; Fernández, C.; Gil, E. y Llopis, J.J. (2001). Vida social de la cocaína. Monografía cocaína. Adicciones. Vol 13, suplemento 2, pp 61-104.

Dean, R.A.; Christian, C.D.; Sample, R.H.B. and Bosron W.F. (1991). Human liver cocaine esterases: Ethanol-mediated formation of ethylcocaine. FASEB J 5: 2735-2739.

Dean, R.A.; Bosron, W.F.; Zachman, F.M. and Brzezinski, M.R. (1997.) Effects of ethanol on cocaine metabolism and disposition in the rat. NIDA Research monograph 173: 35-47.

Farré, M.; de la Torre, R.; Llorente, M.; Lamas, X.; Ugena, B.; Segura, J. and Camí, J. (1993). Alcohol and cocaine interactions in humans. J Pharmacol Exp Ther 266: 1364-1373.

Hayase, T.; Abiru, H.; Yamamoto, Y.; Yamamoto, K. and Fukui, Y. (1998). Brain _-Endorphin inmumoreactivity as an index of cocaine and combined cocaine-ethanol toxicities. Pharmacology Biochemistry and Behavior 60: 263270.

Hearn, W.L.; Flynn, D.D.; Hime, G.W.; Rose, S.; Confino, J.C; Mantero-Atienza, E.; Wetli, C.W. and Mash, D.C. (1991). Cocaethylene: A unique cocaine metabolite displays high affinity for the dopamine transporter. J Neurochem 56: 698-701.

Horger, B.A.; Taylor, J.R.; Elsworth, J.D.; Jatlow, P.I.; and Roth, R.H. (1996). Sensitization to the locomotor activating effects of cocaine following cocaethylene-preexposure. Brain Res 733: 133-137.

Iyer, R.N.; Nobiletti, J.B.; Jatlow, P.I. and Bradberry, C.W. (1995). Cocaine and cocaethylene: effects on extraceIlular dopamine in the primate. Psychopharmacology 120: 105-110.

Jover, R.; Ponsoda, X.; Gómez-Lechón, M.J.; Herrero, C.; del Pino, J. and Castell J.V. (1991). Potentiaton of cocaine hepatotoxicity by ethanol in human hepatocytes. Toxicol Appl Pharmacol 107: 526-534.

Jatlow, P.; McCance, E.F.; Bradberry, C.W.; Elsworth, J.D.; Taylor, J.R. and Roth, R.H. (1996). Alcohol plus cocaine: the whole is more than the sum of its parts. Ther Drug Monit 18: 460-464.
Landry, M.J. (1992). An overview of cocaethylene, an alcohol-derived, psychoactive, cocaine metabolite. J Psychoactive Drugs 24: 273-276.

Lindholm, S.; Rosin, A.; Dahlin, I.; Georgieva, J. and Franck, J. (2001). Ethanol administation potentiates cocaineinduced dopamine levels in the rat nucleus accumbens. Brain Research 915: 176-184.

McCance-Katz, E.F.; Price, L.H..; McDougle, C.J.; Marek, G.J.; Kosten, T.R. and Jatlow, P. (1991). Cocaethylene formation following sequential administration of cocaine and ethanol to humans: Pharmacological, phsiological, and behavioral studies. Soc Neurosci Abs 17: 890.

O.E.D.T. (2001) Informe anual sobre el problema de la drogodependencia en la Unión Europea 2001. Oficina de publicaciones oficiales de las Comunidades Europeas. Luxemburgo.

Pan, W.J. and Hedaya, M.A. (1999). Cocaine and alcohol interactions in the rat: effect of cocaine and alcohol pretreatments on cocaine pharmacokinetics and pharmacodynamics. J Pharma Sci 88(12): 1266-1274.

Perez-Reyes, M. and Jeffcoat, A.R. (1992). Ethanol/cocaine and coaethylene concentrations and their relationship to subjetive and cardiovascular effects. Life Sci 51: 553563.

Perez-Reyes, M. (1994). The order of drug administration: its effects on the interactio between cocaiene and ethanol. Life Sci 55: 541-550.

Pirozhkov, S. V.; Watson, R. R. and Chen, G. (1993). Ethanol enhances inmunosuppression induced by cocaine. Alcohol. Alcohol Suppl 2: 75-82.

P.N.D. (2001). Informe sobre la situación de la Cocaína en España. Abril 2001. Delegación del Gobierno para el Plan Nacional sobre Drogas. PNSD/Internet. Ministerio de Interior

Prinssen, E.P.; Kleven, M.S. and Koek, W. (1996). Repeated administration of cocaethylene induces context-dependent sensitization to its locomotor effects. Psychopharmacology 124: 300-305.

Qiu, Z.; and Morgan, J.P. (1993). Differential effects of cocaine and cocaethylene on intracellular $\mathrm{Ca} 2+$ and myocardial contraction in cardiac myocytes. Br. J. Pharmacol Jun 109: 293-298.

Raven, M. A.; Necessary, B.D.; Danluck, D.A. and Ettenberg, A. (2000) Comparison of the reinforcing and anxiogenic effects of intravenous cocaine and cocaethylene. Exp Clin Psychopharmacol 8: 117-124.

Roberts, S.M.; Roth, L.; Harbison, R.D. and James, R.C. (1992). Cocaethylene hepatotoxicity in mice. Biochem Pharmacol May 8;43: 1989-1995.

Rose, S.; Hearn, W.L.; Hime, G.W.; Wetli, C.V.; Ruttenber, A.J.; and Mash, D.C. (1990). Cocaine and cocaethylene concentrations in human post mortem cerebral cortex. Neurosci Abs 16: 11-17.

Signs, S.A.; Dickey-White, H.I.; Vanek, V.W.; Perch, S.; Schechter, M.D.; and Kulics, A.T. (1996). The formation of cocaethylene and clinical presentation of ED patients testing positive for the use of cocaine and ethanol. Am J Emerg Med Nov 14: 665-670. 
Singer, L.T.; Arendt, R.; Minnes, S.; Farkas, K. and Salvator, A. (2000). Neurobehavioral outcomes of cocaine-exposed infants. Neurotoxicologycal Terotology 22: 653-666.

Schechter, M.D. (1995). Cocaethylene produces conditioned place preference in rats. Pharmacol Biochem Behav 51: 549-552.

Schechter, M.D. and Meehan, S.M. (1995). The lethal effects of ethanol and cocaine and their combinations in mice: implications for cocaethylene formation. Pharmacol Biochem Behav 52: 245-248.

Spealman, R.D.; Madras, B.K. and Bergman, J. (1989). Effects of cocaine and related drugs in nonhuman primates. II. Stimulant effects on schedule-controlled behavior. J Pharmacol Exp Ther 252: 142-149.

Torres, G; Horowitz, J.M.; Lee, S. and Rivier, C. (1996). Cocaethylene stimulates the secretion of $\mathrm{ACTH}$ and corti- costerone and the transcriprional activation of hypothalamic NGFI-B. Brain Res Mol Brain 43: 225-232.

Vanek, V.W.; Dickey-White, H.I.; Schechter, M.D.; Buss, T. and Kulics, A.T. (1996). Concurrent use of cocaine and alcohol by patients treated in the emergency deparment. Ann Emerg Med, Nov 28: 508-514.

Wilson, L.D.; Jeromin, J.; Garvey, L. and Dorbandt, A. (2001). Cocaine, ethanol, and cocaethylene cardiotoxity in an animal model of cocaine and ethanol abuse. Acad Emerg Med 8: 211-222.

Xu, Y. Q.; Crumb, W. J. and Clarkson, C. W. (1994). Cocaethylene a metabolite of cocaine and ethanol, is a potent blocker of cardiac sodium channels. J Pharmacol ExpTher 27: 319-325. 